\title{
Varicella zoster virus (VZV)-specific immunity and subclinical VZV reactivation in patients with autoimmune diseases
}

\author{
Kwang-Hoon Lee ${ }^{1}$, Sungim Choi ${ }^{2}$, Ji-Soo Kwon ${ }^{3}$, Sung-Han Kim³ ${ }^{3}$ and Seong Yeon Park ${ }^{2}$
}

Divisions of ${ }^{1}$ Rheumatology and ${ }^{2}$ Infectious Diseases, Department of Internal Medicine, Dongguk University Ilsan Hospital, Goyang; ${ }^{3}$ Department of Infectious Diseases, Asan Medical Center, University of Ulsan College of Medicine, Seoul, Korea

Received: December 15, 2020

Revised : March 11, 2021 Accepted: March 25, 2021

\section{Correspondence to}

Seong Yeon Park, M.D.

Division of Infectious Diseases, Department of Internal

Medicine, Dongguk University Ilsan Hospital, 27 Dongguk-ro, Ilsandong-gu, Goyang 10326,

Korea

Tel: +82-31-961-7140

Fax: +82-31-961-8331

E-mail: psy99ch@hanmail.net https://orcid.org/0000-0001$8762-7006$
Background/Aims: The risk of herpes zoster (HZ) is increased in patients with autoimmune diseases (AID), probably due to immunosuppressive therapy.

Methods: This prospective cross-sectional study investigated varicella zoster virus (VZV)-specific immunity in relation to subclinical VZV reactivation in 48 AID patients and 48 healthy controls (HCs). We assessed humoral immunity (serum VZV immunoglobulin $\mathrm{g}[\mathrm{IgG}], \mathrm{IgA}$, and IgM) and cell-mediated immunity (interferon- $\gamma$ [IFN $\gamma]$-releasing assay) to VZV as well as salivary VZV DNA status. Subclinical VZV reactivation was confirmed by detecting VZV DNA in saliva or VZV IgM in serum in the absence of typical HZ symptoms.

Results: Median IgA levels were higher in the AID group than in the HC group, while VZV IgG and IgM levels were comparable between the groups. AID patients showed fewer IFN $\gamma$ spot-forming cells (SFCs) upon VZV stimulation than HCs (58.2 vs. $122.0 \mathrm{SFCs} / 10^{6}$ peripheral blood mononuclear cells $[\mathrm{PBMCs}], p<0.0001$ ). Subclinical VZV reactivation was more frequent in AID patients than in HCs $12.5 \%$ vs. $\circ \%, p=0.01$ ). AID patients with VZV reactivation received prednisolone more frequently and at a higher dose than AID patients without reactivation. VZV-specific IFN $\gamma$ SFCs were significantly lower in patients with VZV reactivation among AID patients (26.3 vs. $62.6 \mathrm{SFCs} / 10^{6} \mathrm{PBMCs}, p<0.0001$ ).

Conclusions: Results suggest that poor cellular response against VZV might cause clinical and subclinical reactivation of VZV in AID patients.

Keywords: Varicella zoster virus; Autoimmune diseases; Subclinical reactivation; Immunity, cellular

\section{INTRODUCTION}

The varicella zoster virus (VZV) is a ubiquitous human alpha-herpesvirus. The virus becomes latent in neurons of the dorsal root ganglia, cranial nerve ganglia, and autonomic ganglia after primary infection. Although the mechanism of reactivation is not fully understood, it is likely that reactivation may occur when VZV-specific cell-mediated immunity (CMI) decreases below a threshold level [1]. Reactivation from latency causes herpes zoster (HZ), which is a localized, painful vesicular rash that usually appears unilaterally in the distribution of one or more adjacent sensory nerves.

The highest risk of reactivation is observed in patients with inflammatory myositis and in those with systemic lupus erythematosus (SLE), irrespective of age $[2,3]$. The prevalence of $\mathrm{HZ}$ is estimated to be 15 to 91 cases per 1,000 person-years among SLE patients $[4,5]$ and 12 cases 
per 1000 person-years among patients with rheumatoid arthritis (RA) [6]. The above statistics imply that there is at least a 4 - to 20 -fold increase in HZ risk among SLE and RA patients, compared to that in the general population. HZ rash can be prolonged, severe, or disseminated in patients with autoimmune disease (AID). The risk of severe neurologic complications such as myelitis, chronic encephalitis, and meningoencephalitis increases further in this population [7]. Moreover, in patients with AID, incident HZ was associated with a 2 -fold increase in the risk of stroke subsequent to HZ occurrence $[8,9]$. Thus, prevention of HZ is critical in this population. In this context, guidelines that include recommendations for the use of $\mathrm{HZ}$ vaccination in patients with AID are not entirely consistent with one another [10,11], and, in fact, SLE patients show the lowest HZ vaccination rates among the age-eligible subjects [3]. More knowledge of the underlying immunity to VZV is needed to understand the increased susceptibility of AID patients to HZ to support HZ prevention.

It is already known that VZV-specific CMI is reduced in patients with AID because of the use of immunosuppressive therapy, leading not only to clinical reactivation of VZV (HZ) [12-15], but also to subclinical VZV reactivation. However, little is known about subclinical VZV reactivation in this population. To our knowledge, there is only one report regarding VZV subclinical reactivation in SLE [16]. Thus, we compared VZV-specific immunity and subclinical VZV reactivation between patients with AID and healthy controls (HCs) to test whether decreased CMI might cause subclinical reactivation.

\section{METHODS}

\section{Study population}

We enrolled patients with AID aged $\geq 18$ years who visited the Dongguk University Ilsan Hospital outpatient clinic as well as age-matched HCs, between March 2018 and April 2019. HCs were recruited as volunteers during the same study period. Patients with RA, SLE, dermatomyositis, polymyalgia rheumatica, and mixed connective tissue diseases were included in the AID group. RA patients who fulfilled the American College of Rheumatology (ACR)/European League Against Rheumatism (EULAR) criteria [7] were eligible for inclusion. AIDs other than RA were diagnosed using the appropriate diagnostic criteria. Disease characteristics, disease activity, and data on the use of immunosuppressive therapies were recorded. Comprehensive data on vaccination and HZ history were obtained from patient medical records, vaccination documents, or by interviewing the patients. Disease activity of RA was determined by calculating the Disease Activity Score 28-erythrocyte sedimentation rate (DAS28-ESR) [17] and ESR and by measuring C-reactive protein (CRP) levels. Patients who had experienced active HZ or had been vaccinated before sample acquisition were excluded from the study. This study complies with the Declaration of Helsinki, and the protocols of this study were approved by the Institutional Review Board of the Dongguk University Ilsan Hospital (IRB no. 2017-11-002). Informed consents were obtained from all patients and control subjects.

\section{Antibody response to VZV and diphtheria}

Anti-VZV immunoglobulin g (IgG), IgM, and IgA antibodies were detected using glycoprotein ELISA assays (Institut Virion/Serion GmbH, Würzburg, Germany) according to the manufacturer's protocol. In brief, $100 \mu \mathrm{L}$ of controls and diluted plasma samples were added to a pre-coated 96-well plate and incubated for 1 hour. After a washing step, we added $100 \mu \mathrm{L}$ of goat anti-human IgG, IgM, or IgA conjugated with alkaline phosphatase, and the plate was incubated for 30 minutes. After a further washing step, $100 \mu \mathrm{L}$ of substrate solution was added, and the enzymatic reaction was quantified after $30 \mathrm{~min}$ utes of incubation. All incubation steps were performed at $37^{\circ} \mathrm{C}$. Data were acquired using a Spark microplate reader (Tecan Trading AG, Männedorf, Switzerland). Because there is evidence of polyclonal hypergammaglobulinemia in SLE patients, IgG antibody responses to diphtheria were included as controls and measured using a commercial kit according to the manufacturer's instructions (Institut Virion/Serion GmbH). Diphtheria was chosen because it is nonendemic in South Korea, as previously reported [18].

\section{VZV-specific CMI}

CMI to VZV was evaluated using the interferon- $\gamma$ (IFN $\gamma$ )-release assay [19]. Peripheral blood mononuclear cells (PBMCs) in heparinized blood were separated by Ficoll-Hypaque density gradient centrifugation (Sig- 
ma-Aldrich, St. Louis, MO, USA) and subsequently frozen. Previously cryopreserved PBMCs with a viability of $\geq 70 \%$ were added at 200,000 cells/well were stimulated in quadruple wells with VZV grade 2 antigen (EL-03o2 Microbix, Mississauga, ON, USA), and with Medical Research Council cell strain 5 (MRC5) control antigen (Microbix ELC-29-02) as a negative control, or phytohemagglutinin as a positive control. After overnight incubation, spot-forming cells (SFCs) were counted using an AIDiSpot (Autoimmune diagnostika GmbH, Straßberg, Germany) instrument. Results were reported as the number of SFCs $/ 10^{6}$ PBMCs in VZV-stimulated wells after subtracting the number of SFCs in control wells containing MRC5.

\section{VZV-specific polymerase chain reaction assays using saliva}

To examine subclinical reactivation of VZV in saliva, saliva samples were collected at least twice every month. Saliva samples (1 mL or more) were collected with an Omnigene-Oral kit (DNA Genotek, Ottawa, ON, Canada) at any time of the day but at least 1 hour after a meal. The samples were shaken vigorously for at least $10 \mathrm{sec}-$ onds and incubated in a water bath at $50^{\circ} \mathrm{C}$ for 1 hour. DNA was extracted with a QiAamp DNA mini kit (Qiagen Inc., Chatsworth, CA, USA) as recommended by the manufacturer. VZV was quantified with a VZV real-time polymerase chain reaction (PCR) kit (GeneProof, Brno, Czech Republic) using a LightCycler 480 System (Roche, Basel, Switzerland). A specific conserved DNA sequence of the ORF62 gene was used as the target sequence. The copy number of VZV DNA was determined by comparing the cycle threshold values of the test samples with those of the reference VZV DNA supplied with the PCR kit $[19,20]$.

\section{Subclinical reactivation of VZV}

In line with the definition of Rondaan et al. [16], subclinical reactivation of VZV was defined as the presence of VZV DNA in saliva or a positive VZV IgM status (unless explained by the presence of rheumatoid factor), in the absence of clinical signs or symptoms of HZ or chickenpox. VZV IgM positivity was defined as a VZV IgM level of more than $100 \mathrm{IU} / \mathrm{mL}$, as per the manufacturer's recommendation. We also analysed VZV IgA since it has been helpful for HZ diagnosis [21,22] although it has not been included it in the definition of VZV reactivation by Rondaan et al. [16].

\section{Statistical analysis}

IBM SPSS statistics for Windows version 20.0 (IBM Corp., Armonk, NY, USA) was used for all statistical analyses. Graphs were constructed using GraphPad Prism 8 for Windows (GraphPad Software Inc., La Jolla, CA, USA). Continuous variables are expressed as median values with interquartile ranges (IQRs) and are also compared with the Mann-Whitney $U$ test. Categorical variables were expressed as numbers and percentages and analysed using the chi-square test. Two-tailed $p$ values $<0.05$ were considered statistically significant.

\section{RESULTS}

\section{Baseline characteristics}

A total of 96 subjects, including 48 HCs and 48 patients with AID ( 35 RA, seven SLE, three dermatomyositis, one adult-onset Still's disease, one polymyalgia rheumatica, and one mixed connective tissue disease) were prospectively enrolled during the study period. The demographic characteristics of the participants are shown in Table 1. There were no significant differences in age between the AID and HC groups, while more women were included in the AID group. A majority of RA patients (34/35) were taking anti-rheumatic drugs, including methotrexate and biologic agents, at the time of study inclusion. Disease activity was relatively low (median DAS28-ESR 2.47 [IQR, 2.28 to 3.05], ESR 19.0 mm/hr [IQR, 10.5 to 28.0 ], and CRP level $0.08 \mathrm{mg} / \mathrm{dL}$ [IQR, 0.04 to 0.32]). Seven patients with SLE received hydroxychloroquine, mycophenolate mofetil (MMF), or prednisolone therapy. All patients in the AID group were examined by a rheumatologist (K.H.L.) at the study hospital. The median disease duration was 36.0 months (IQR, 11 to 70 ).

\section{VZV and diphtheria antibody levels.}

All study subjects tested positive for VZV IgG. The levels of VZV IgG antibodies were not significantly different between the AID (median, 543.7 IU/mL [IQR, 326.1 to 938.0]) and $\mathrm{HC}(694.8 \mathrm{IU} / \mathrm{mL}$ [IQR, 337.4 to 1,158$])(p$ $=0.28$ ) groups (Fig. 1A). However, the levels of VZV IgA antibodies in the AID group were significantly higher 
Table 1. Baseline characteristics of study participants

\begin{tabular}{|c|c|c|c|}
\hline Characteristic & $\begin{array}{l}\text { Healthy controls } \\
\qquad(\mathrm{n}=48)\end{array}$ & $\begin{array}{l}\text { Autoimmune disease patients } \\
\qquad(\mathrm{n}=48)\end{array}$ & $p$ value \\
\hline Age, yr & $50.0(43.3-58.8)$ & $49.0(42.3-58.0)$ & 0.51 \\
\hline Female sex & $28(58.3)$ & $38(79.2)$ & 0.02 \\
\hline \multicolumn{4}{|l|}{ Distribution of disease } \\
\hline RA & & $35(72.9)$ & \\
\hline SLE & & $7(14 \cdot 6)$ & \\
\hline Dermatomyositis & & $3(6.3)$ & \\
\hline Others $^{\mathrm{a}}$ & & $3(6.3)$ & \\
\hline Treatment & NA & & \\
\hline Prednisolone & & $27(56.3)$ & \\
\hline Dose, mg/day & & $5.0(1.25-15.0)$ & \\
\hline Methotrexate & & $36(75 \cdot 0)$ & \\
\hline Dose, mg/week & & $15.0(10.0-15.0)$ & \\
\hline Hydroxychloroquine & & $6(12.5)$ & \\
\hline Mycophenolate mofetile & & $3(6.3)$ & \\
\hline Biologic agent & & $9(18.8)$ & \\
\hline Tocilizumab & & $3(8.6)$ & \\
\hline Golimumab & & $3(8.6)$ & \\
\hline Tofacitinib & & $2(5.7)$ & \\
\hline Infliximab & & $1(2.9)$ & \\
\hline Disease duration, mo & NA & $36(11-70)$ & \\
\hline
\end{tabular}

Values are presented as median (interquartile range) or number (\%).

RA, rheumatoid arthritis; SLE, systemic lupus erythematous; NA, not available.

${ }^{a}$ Others include one adult onset still's disease, one polymyalgia rheumatic, and one mixed connective tissue disease.

than those in the HC group (3.04 IU/mL vs. $1.16 \mathrm{IU} / \mathrm{mL}$, $p=0.02$ ) (Fig. 1B). No significant differences were found in IgM antibody levels between the AID and HC groups (Fig. ${ }_{1 C}$ ). In contrast to VZV IgG and IgM, Diphtheria antibody levels in AID patients were significantly lower than in $\mathrm{HCs}(p=0.02)$ (Fig. 1D).

\section{VZV specific CMI}

In the IFN $\gamma$ enzyme-linked immunosorbent assay, the number of SFCs observed in response to VZV stimulation was significantly lower in AID patients than in HCs (median number of IFN $\gamma$ SFCs/10 $10^{6}$ PBMCs: 58.2 [IQR, 45.0 to 70.0] vs. 122.0 [IQR, 108.1 to 188.1], $p<0.001$ ) (Fig. 2). In a subgroup analysis that sorted patients with AID according to the type of AID (RA, SLE, or other AID), the number of IFN $\gamma$ SFCs $/ 10^{6}$ PBMCs was not significantly different among these subgroups (RA vs. SLE vs. other
AID: 60.0 [IQR, 49.0 to 75.0] vs. 54.6 [IQR, 31.5 to 69.0] vs. 50.5 [IQR, 24.9 to 79.8], $p=0.46$ ).

\section{Subclinical reactivation of VZV}

Subclinical VZV reactivation was detected in six AID patients by PCR for VZV DNA, and VZV IgM positivity was detected in none of the study patients. No reactivation was observed in the HC group. Subclinical VZV reactivation was significantly more frequent in patients with AID than in HCs (12.5\% vs. $0 \%, p=0.01)$ and occurred in two RA patients, two patients with SLE, one patient with dermatomyositis, and one patient with adult-onset Still's disease. Among the six patients with positivity for salivary VZV DNA, the median viral load was 2.32 log copies/mL (IQR, 1.75 to 3.45). We compared the clinical characteristics of 48 AID patients with and without subclinical VZV reactivation (Table 2). Interestingly, pred- 

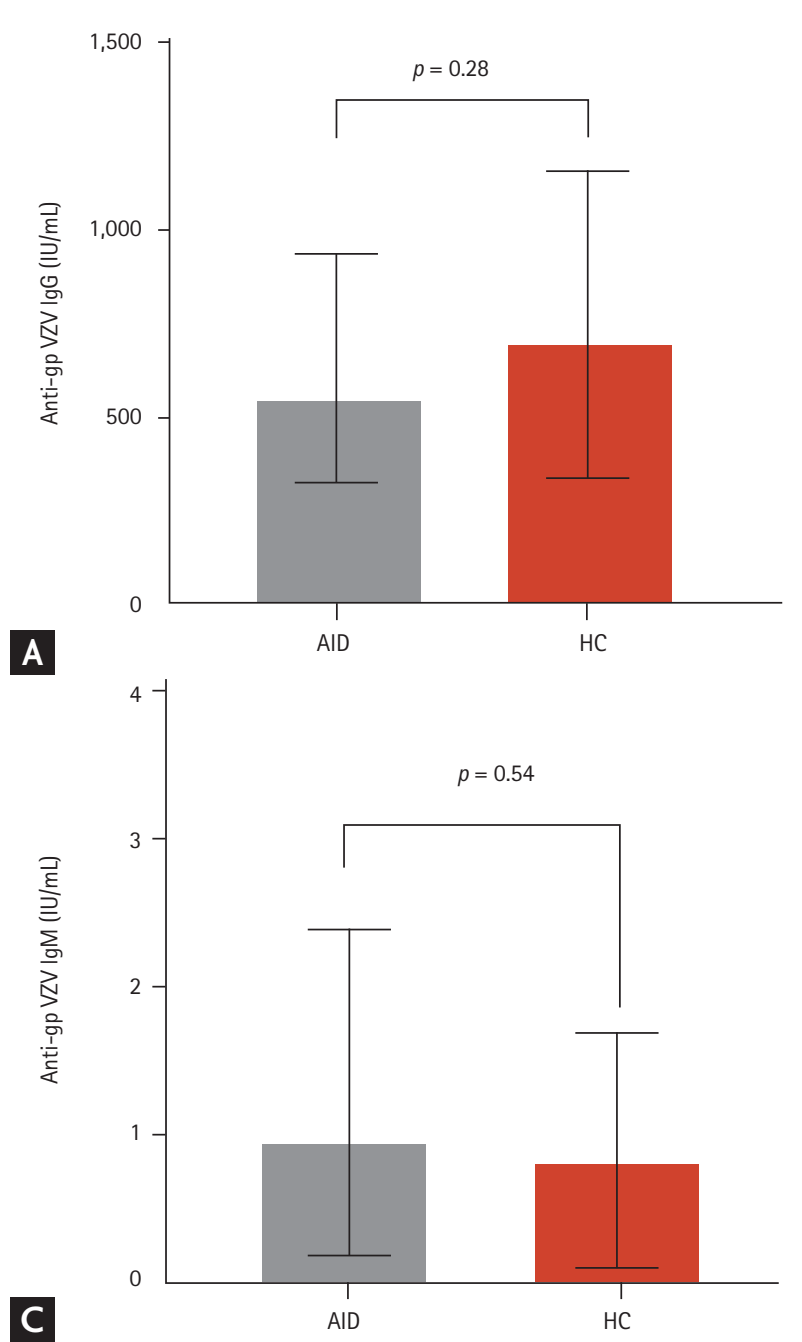

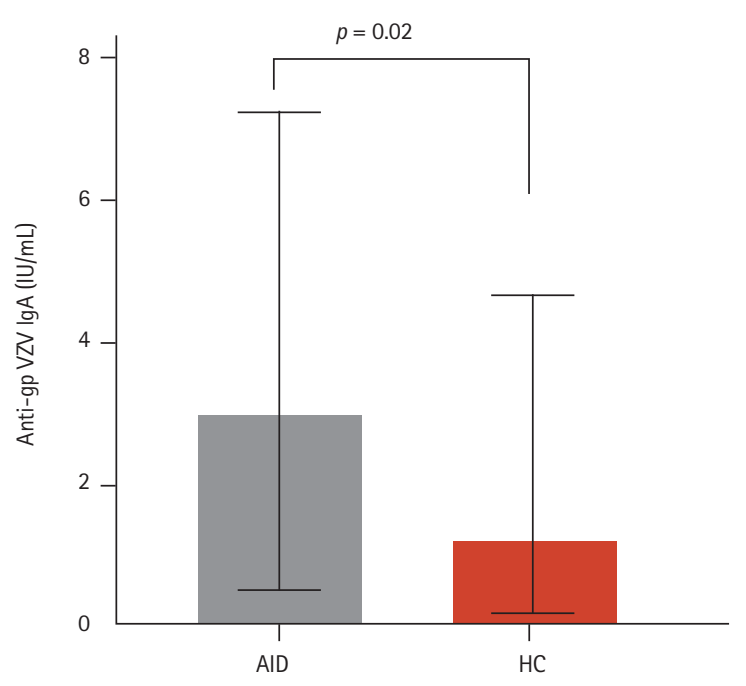

B

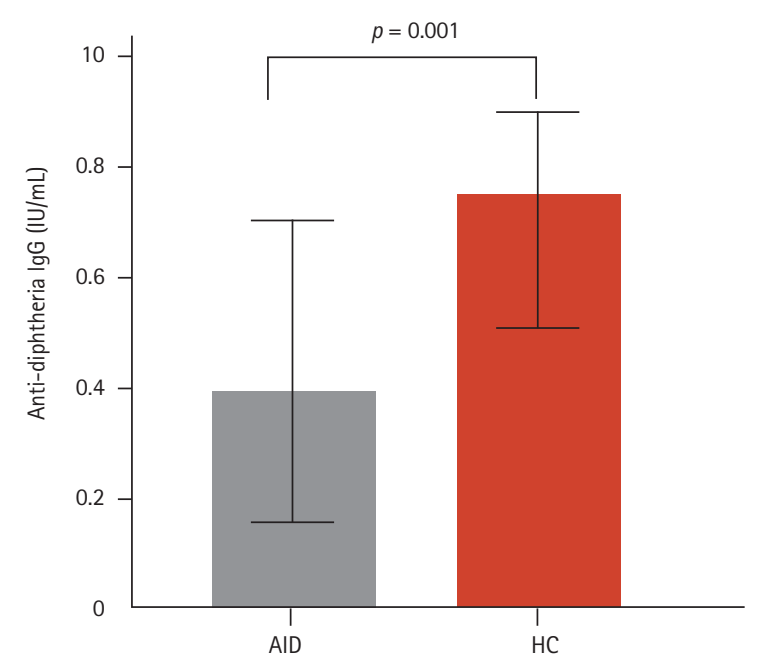

Figure 1. Levels of antibodies against varicella zoster virus (VZV) and diphtheria. Bars show the median and interquartile range. (A) Anti-gpVZV immunoglobulin g (IgG), (B) anti-gpVZV IgA, (C) anti-gpVZV IgM, (D) anti-diphtheria IgG. gp, glycoprotein; AID, autoimmune disease; HC, healthy control.

nisolone use (100\% vs. $50.0 \%, p=0.02)$ as well as the median prednisolone dose (15.0 mg/day vs. $2.5 \mathrm{mg} /$ day, $p=$ o.o1) were higher in patients with VZV reactivation than in those without VZV reactivation. In addition, the median number of VZV-specific IFN $\gamma$ SFCs was significantly lower in patients with VZV reactivation than in those without VZV reactivation (26.3 SFCs $/ 10^{6}$ PBMCs vs. 62.6 SFCs $/ 10^{6}$ PBMCs, $p<0.0001$ ).

\section{DISCUSSION}

In this study, we found decreased VZV-specific CMI in
AID patients compared to HCs. However, humoral immunity to VZV was not impaired in the AID group. Subclinical reactivation of VZV was frequently observed in AID patients but not in HCs. In addition, VZV-specific CMI was significantly lower in patients with subclinical VZV reactivation among AID patients.

Since VZV-specific T-cells have been shown to be important for effective VZV control, VZV reactivation may occur when VZV-specific CMI falls below a critical threshold level [1]. AID patients have impaired VZV-specific CMI owing to the immunosuppressive effects of the disease and/or the use of immunomodulatory medication [12-15,18]. In the current study, the majority of 
participants in the AID group (72.9\%) were RA patients. A previous study reported that VZV-specific CD4 T-cell levels were lower in patients with RA than in HCs [12]; treatment regimens, such as those involving biological disease-modifying anti-rheumatic drugs, have been re-

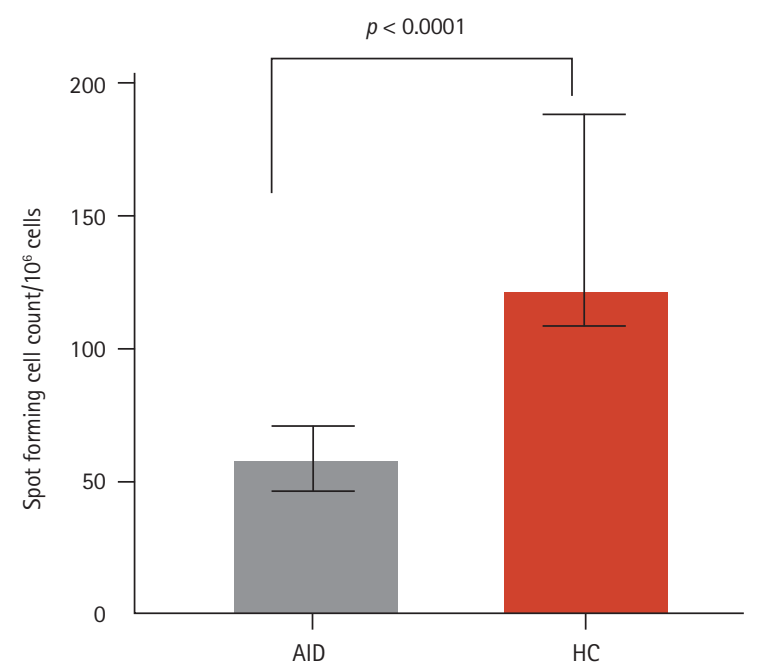

Figure 2. Levels of interferon- $\gamma$ (IFN $\gamma$ ) spot-forming cells in response to varicella zoster virus (VZV) stimulation. Numbers of IFN $\gamma$ spot-forming cells $/ 10^{6}$ peripheral blood mononuclear cells in rheumatoid arthritis patients and healthy controls (HCs) in response to VZV stimulation were determined. Bars show the median and interquartile range. AID, autoimmune disease. ported to attenuate cellular immunity [14]. IFN $\gamma$ plays an important role in the host immune response to viral infections. Methotrexate has been shown to suppress IFN- $\gamma$, which may result in VZV reactivation [23]. Moreover, a dose-dependent association between the use of corticosteroids and HZ risk has been reported [8]. In addition to RA patients, decreased CMI was observed in patients with SLE $[15,18]$. The use of prednisolone and MMF in SLE poses additional risks for HZ [3]. In line with previous reports $[12-15,18]$, these findings might suggest an increased susceptibility to VZV reactivation in patients with AID.

Despite the absence of clinical symptoms, subclinical VZV reactivation was frequently observed in patients with AID (12.5\% vs. $0 \%, p=0.01)$. Patients who had e xperienced VZV reactivation showed VZV DNA in their saliva $[19,20,24]$. AID patients may be susceptible to VZV reactivation for a number of reasons. First, a transient decrease in normal immune function associated with increased blood cortisol levels was experienced by astronauts during space flight, as demonstrated by scientists at the National Aeronautics and Space Administration $[25,26]$. Although we could not measure cortisol levels in the study population, we believe that stress may induce subclinical reactivation in the absence of clinical disease [25-27]. AID flares might also lead to subclini-

Table 2. Comparison of patients with and without subclinical VZV reactivation in patients with autoimmune disease

\begin{tabular}{lccc}
\hline Variable & $\begin{array}{c}\text { VZV reactivation } \\
(\mathrm{n}=6)\end{array}$ & $\begin{array}{c}\text { Without VZV reactivation } \\
(\mathrm{n}=42)\end{array}$ & $p$ value \\
\hline Age & $41.5(29.8-47.0)$ & $50.5(44.0-59.8)$ & 0.10 \\
Female sex & $5(83.3)$ & $33(78.6)$ & 0.63 \\
Treatment & $3(50.0)$ & $33(78.6)$ & 0.16 \\
Methotrexate & $6(100)$ & $21(50.0)$ & 0.02 \\
Prednisolone & $15.0(8.8-25.0)$ & $2.5(1.25-7.5)$ & 0.01 \\
Median PD dose, mg/day & $1(16.7)$ & $8(19.0)$ & 0.67 \\
Biologic agent & & $2.43(1.98-23.0)$ & 0.24 \\
VZV specific immunity & $6.99(2.0-23.0)$ & $543.7(328.2-970.3)$ & 0.68 \\
VZV IgA & $488.1(165.4-1,462.1)$ & $0.94(0.19-2.39)$ & 0.74 \\
VZV IgG & $1.11(0.22-6.72)$ & $62.6(50.0-76.8)$ & $<0.01$ \\
VZV IgM & $26.3(14.4-33.5)$ & &
\end{tabular}

Values are presented as median (interquartile range) or number (\%).

VZV, varicella zoster virus; PD, prednisolone; Ig, immunoglobulin; IFN $\gamma$, interferon- $\gamma$; SFC, spot-forming cell. 
cal virus reactivation. Second, subclinical VZV viremia was observed in individuals with malignancies, and virus-specific T-cell responses were the lowest in those with subclinical virus reactivation [28]. In this study, patients with subclinical VZV reactivation had significantly lower VZV-specific CMI than patients without VZV reactivation (26.3 SFCs $/ 10^{6} \mathrm{PBMCs}$ vs. $62.6 \mathrm{SFCs} / 10^{6}$ PBMCs, $p<0.0001)$. This finding suggests that VZV-specific CMI could be important for controlling VZV reactivation. Another interesting finding is that subclinical VZV reactivation is associated with prednisolone use. Patients with VZV reactivation took prednisolone more frequently (100\% vs. 50.0\%, $p=0.02)$ and at a higher median dose $(15.0 \mathrm{mg} /$ day vs. $2.5 \mathrm{mg} /$ day, $p=0.01)$ than patients without VZV reactivation. Steroid effects have been well demonstrated for herpes simplex virus, another alpha-herpesvirus. Harrison et al. [29] reported that dexamethasone stimulated explant-induced HSV reactivation from latency but, conversely, a glucocorticoid receptor antagonist significantly impaired reactivation from latency. However, this has never been shown in VZV.

In this study, humoral immunity to VZV was comparable between the AID and HC groups. This is in line with the results of a previous study [12] and supports the assumption that poor VZV-specific CMI, but not humoral immunity, may be the main contributor to VZV reactivation. However, other studies have reported significantly higher VZV IgG levels in SLE patients [18] and lower VZV IgG levels in RA patients compared to those in a matched control group without RA [13,14]. Meanwhile, level of VZV IgG can be boosted during subclinical reactivation or exposure to $\mathrm{VZV}$, which can be one of explanations why the level of VZV IgG was similar between AID patients and HCs. Evidence suggests that the endogenous boosting of VZV antibody levels after clinical VZV reactivation and in the absence of $\mathrm{HZ}$ are associated with the subclinical VZV reactivation or re-exposure to VZV [21,30].

Interestingly, VZV IgA was higher in AID patients. $\operatorname{IgA}$, as the principal antibody class in the secretions that bathe these mucosal surfaces, acts as an important first line of defense [31]. Because VZV is presumed to have a respiratory mode of transmission, antibodies of the IgA class may be important in the host response. VZV-specific IgA has been suggested to have some value in the diagnosis of HZ and chickenpox [21,22,32]. Level of VZV IgA might be boosted during subclinical reactivation or exposure to VZV in this study. However, the clinical significance of subclinical reactivation is unclear. Thus, further research is needed to clarify VZV-specific humoral immunity in this population.

A limitation of our study is that the sample size was relatively small, and the AID patient group was heterogeneous. Additionally, follow-up data to assess changes in the quantitative levels of specific CMI and humoral immunity markers in relation to clinical reactivation events were unavailable. Thus, longitudinal studies involving large sample sizes are needed to confirm these results. Our study suggests that the use of steroids decreases VZV-specific CMI and, as a result, subclinical reactivation was frequently observed in AID patients. However, we did not demonstrate the effect of steroids on VZV-infected cell growth or VZV-specific CMI in vitro. Therefore, further studies using a cell model such as fibroblast infected cell lines are needed to show if steroid treatment affects VZV spread.

In conclusion, patients with AID showed decreased CMI against VZV, while humoral immunity was not impaired in these patients. Subclinical VZV reactivation was observed in patients with AID. These data suggest that the increased susceptibility to clinical or subclinical VZV reactivation in patients with AID may be due to a poor cellular response. Vaccination strategies should aim to boost cellular immunity against VZV.

\section{KEY MESSAGE}

1. The risk of herpes zoster is increased in patients with autoimmune diseases (AID); however, little is known about subclinical reactivation in this population.

2. Thus, we compared varicella zoster virus (VZV)-specific humoral and cellular immunity in relation to subclinical VZV reactivation between patients with AID and healthy controls (HCs) whether decreased cell-mediated immunity (CMI) might cause subclinical reactivation.

3. VZV-specific CMI was decreased in AID patients and subclinical reactivation of VZV was 
frequently observed in AID patients but not in HCs. In addition, VZV-specific CMI was significantly lower in patients with subclinical VZV reactivation among AID patients. Results suggest that poor cellular response against VZV might cause clinical and subclinical reactivation of VZV in AID patients.

\section{Conflict of interest}

No potential conflict of interest relevant to this article was reported.

\section{Acknowledgments}

This study was supported by a National Research Foundation of Korea (NRF) grant funded by the Korean government (MSIP) (grant no. NRF-2017R1C1B2003336). This study was also supported by the Korean Society of Internal Medicine (Grant 2017).

\section{REFERENCES}

1. Arvin A. Aging, immunity, and the varicella-zoster virus. N Engl J Med 2005;352:2266-2267.

2. Yun H, Yang S, Chen L, et al. Risk of herpes zoster in autoimmune and inflammatory diseases: implications for vaccination. Arthritis Rheumatol 2016;68:2328-2337.

3. Chakravarty EF, Michaud K, Katz R, Wolfe F. Increased incidence of herpes zoster among patients with systemic lupus erythematosus. Lupus 2013;22:238-244.

4. Bond D, Mooney J. A literature review regarding the management of varicella-zoster virus. Musculoskeletal Care 2010;8:118-122.

5. Chen HH, Chen YM, Chen TJ, Lan JL, Lin CH, Chen DY. Risk of herpes zoster in patients with systemic lupus erythematosus: a three-year follow-up study using a nationwide population-based cohort. Clinics (Sao Paulo) 2011;66:1177-1182.

6. Veetil BM, Myasoedova E, Matteson EL, Gabriel SE, Green $\mathrm{AB}$, Crowson CS. Incidence and time trends of herpes zoster in rheumatoid arthritis: a population-based cohort study. Arthritis Care Res (Hoboken) 2013;65:854-861.

7. Aletaha D, Neogi T, Silman AJ, et al. 2010 Rheumatoid arthritis classification criteria: an American College of
Rheumatology/European League Against Rheumatism collaborative initiative. Arthritis Rheum 2010;62:25692581.

8. Liao TL, Lin CH, Chen HH, Chen YM, Lin CC, Chen DY. significant associations of neurological complications of herpes zoster with stroke in rheumatoid arthritis patients. J Am Heart Assoc 2017;6:e006304.

9. Calabrese LH, Xie F, Yun H, et al. Herpes zoster and the risk of stroke in patients with autoimmune diseases. Arthritis Rheumatol 2017;69:439-446.

10. Furer V, Rondaan C, Heijstek MW, et al. 2019 Update of EULAR recommendations for vaccination in adult patients with autoimmune inflammatory rheumatic diseases. Ann Rheum Dis 2020;79:39-52.

11. Singh JA, Furst DE, Bharat A, et al. 2012 Update of the 2008 American College of Rheumatology recommendations for the use of disease-modifying antirheumatic drugs and biologic agents in the treatment of rheumatoid arthritis. Arthritis Care Res (Hoboken) 2012;64:625639.

12. Schub D, Assmann G, Sester U, Sester M, Schmidt T. VZV-specific T-cell levels in patients with rheumatic diseases are reduced and differentially influenced by antirheumatic drugs. Arthritis Res Ther 2018;20:252.

13. Krasselt M, Baerwald C, Liebert UG, Seifert O. Humoral immunity to varicella zoster virus is altered in patients with rheumatoid arthritis. Clin Rheumatol 2019;38:24932500.

14. Almanzar G, Kienle F, Schmalzing M, Maas A, Tony HP, Prelog M. Tofacitinib modulates the VZV-specific CD4+ $\mathrm{T}$ cell immune response in vitro in lymphocytes of patients with rheumatoid arthritis. Rheumatology (Oxford) 2019;58:2051-2060.

15. Park HB, Kim KC, Park JH, et al. Association of reduced $\mathrm{CD}_{4} \mathrm{~T}$ cell responses specific to varicella zoster virus with high incidence of herpes zoster in patients with systemic lupus erythematosus. J Rheumatol 2004;31:2151-2155.

16. Rondaan C, van Leer CC, van Assen S, et al. Longitudinal analysis of varicella-zoster virus-specific antibodies in systemic lupus erythematosus: no association with subclinical viral reactivations or lupus disease activity. Lupus 2018;27:1271-1278.

17. Prevoo ML, van 't Hof MA, Kuper HH, van Leeuwen MA, van de Putte LB, van Riel PL. Modified disease activity scores that include twenty-eight-joint counts. Development and validation in a prospective longitudinal study 
of patients with rheumatoid arthritis. Arthritis Rheum 1995;38:44-48.

18. Rondaan C, de Haan A, Horst G, et al. Altered cellular and humoral immunity to varicella-zoster virus in patients with autoimmune diseases. Arthritis Rheumatol 2014;66:3122-3128.

19. Park SY, Kim JY, Kwon JS, et al. Relationships of varicella zoster virus (VZV)-specific cell-mediated immunity and persistence of VZV DNA in saliva and the development of postherpetic neuralgia in patients with herpes zoster. J Med Virol 2019;91:1995-2000.

20. Park SY, Kim JY, Kim JA, et al. Diagnostic usefulness of varicella-zoster virus real-time polymerase chain reaction analysis of DNA in saliva and plasma specimens from patients with herpes zoster. J Infect Dis 2017;217:51-57.

21. Laing KJ, Ouwendijk WJD, Koelle DM, Verjans GMGM. Immunobiology of varicella-zoster virus infection. J Infect Dis 2018;218(Suppl 2):S68-S74.

22. Schub D, Janssen E, Leyking $\mathrm{S}$, et al. Altered phenotype and functionality of varicella zoster virus-specific cellular immunity in individuals with active infection. J Infect Dis 2015;211:600-612.

23. Neurath MF, Hildner K, Becker C, et al. Methotrexate specifically modulates cytokine production by $\mathrm{T}$ cells and macrophages in murine collagen-induced arthritis (CIA): a mechanism for methotrexate-mediated immunosuppression. Clin Exp Immunol 1999;115:42-55.

24. Mehta SK, Tyring SK, Gilden DH, et al. Varicella-zoster virus in the saliva of patients with herpes zoster. J Infect
Dis 2008;197:654-657.

25. Mehta SK, Cohrs RJ, Forghani B, Zerbe G, Gilden DH, Pierson DL. Stress-induced subclinical reactivation of varicella zoster virus in astronauts. J Med Virol 2004;72:174-179.

26. Rooney BV, Crucian BE, Pierson DL, Laudenslager ML, Mehta SK. Herpes virus reactivation in astronauts during spaceflight and its application on Earth. Front Microbiol 2019;10:16.

27. Uchakin PN, Parish DC, Dane FC, et al. Fatigue in medical residents leads to reactivation of herpes virus latency. Interdiscip Perspect Infect Dis 2011;2011:571340.

28. Malavige GN, Rohanachandra LT, Jones L, et al. IE63-specific T-cell responses associate with control of subclinical varicella zoster virus reactivation in individuals with malignancies. Br J Cancer 2010;102:727-730.

29. Harrison KS, Zhu L, Thunuguntla P, Jones C. Antagonizing the glucocorticoid receptor impairs explant-induced reactivation in mice latently infected with herpes simplex virus 1. J Virol 2019;93:e0o418-eoo419.

30. Warren-Gash C, Forbes H, Maple P, Quinlivan M, Breuer J. Viral load and antibody boosting following herpes zoster diagnosis. J Clin Virol 2018;103:12-15.

31. Woof JM, Kerr MA. The function of immunoglobulin A in immunity. J Pathol 2006;208:270-282.

32. Tovi F, Hadar T, Sidi J, Sarov B, Sarov I. The significance of specific IgA antibodies in the serum in the early diagnosis of zoster. J Infect Dis 1985;152:230. 